\title{
Laterality Preferences in Athletes: Insights from a Database of 1770 Male Athletes
}

\author{
Wei-Chun Wang ${ }^{1}$, Matthew DeLang ${ }^{2}$, Kelly Vittetoe ${ }^{3}$, Benjamin Ramger ${ }^{3}$, Susan Hilbig ${ }^{3,4}$, \\ Lawrence Gregory Appelbaum, ${ }^{3,4}$, \\ ${ }^{1}$ Center for Cognitive Neuroscience, Duke University, Durham, USA \\ ${ }^{2}$ Physical Therapy Department, School of Medicine, Duke University, Durham, USA \\ ${ }^{3}$ Department of Psychiatry and Behavioral Science, Duke University School of Medicine, Durham, USA \\ ${ }^{4}$ Sports Vision Center, Duke University School of Medicine, Durham, USA
}

Email address:

weichunw@gmail.com (Wei-Chun W.), matthew.delang@duke.edu (M. DeLang), kelly.vittetoe@duke.edu (K. Vittetoe), benjamin.ramger@duke.edu (B. Ramger), susan.hilbig@duke.edu (S. Hilbig), greg@duke.edu (L. G. Appelbaum)

${ }^{*}$ Corresponding author

\section{To cite this article:}

Wei-Chun Wang, Matthew DeLang, Kelly Vittetoe, Benjamin Ramger, Susan Hilbig, Lawrence Gregory Appelbaum. Laterality Preferences in Athletes: Insights from a Database of 1770 Male Athletes. American Journal of Sports Science. Vol. 6, No. 1, 2018, pp. 20-25.

doi: 10.11648/j.ajss.20180601.14

Received: December 29, 2017; Accepted: January 30, 2018; Published: February 24, 2018

\begin{abstract}
Laterality preferences are inherent in most sensory and motor activities, and sports are certainly one domain wherein these preferences might impact performance and outcomes. The fact that most individuals exhibit laterality preferences and that sporting demands differentially draw on these abilities makes the expression of these preferences in athletes a topic ripe for exploration. To fill this gap, the current report describes hand, foot, and eye laterality preferences in a large cohort of 1770 male athletes tested on the Nike Sensory Station assessment battery. Self-reported hand and foot preferences, as well as eye dominance measured through the Miles Test, were compared across athletes of different experience levels (middle/high school, college, professional athletes) and primary sports (baseball, basketball, football, hockey, soccer) to evaluate group differences in laterality preferences. Results revealed group differences, most notably a higher proportion of left-hand and left-foot preferences in professional baseball players. These findings offer new insight into the associations among laterality preferences in a large and diverse population of athletes.
\end{abstract}

Keywords: Laterality Preferences, Handedness, Footedness, Eye Dominance, Athletes

\section{Introduction}

The vast majority of individuals exhibit some degree of inherent lateralized body preferences that shape the way they perceive and interact with the world. In the general population, the right side of the body is typically preferred approximately $90 \%$ of the population displays right hand preference, $80 \%$ right foot preference, and $70 \%$ right eye preference [1]. In athletic populations this right-side bias is notably reduced [2], leading to questions and research addressing human performance as well as the evolutionary and selection biases that might contribute to this relative increase in left-sided preferences [3, 4].

With few exceptions, sporting activities are usually free of restrictions specifying use of the left or right, and athletes are free to choose the hand or foot with which they throw, catch, kick, or punch. Some athletes may prefer their right or left side for any number of activities, while others may be equally proficient with both sides. Because many activities require the coordination of body parts on both sides of the body, individual differences may also be expressed as crossed dominance, such as when a baseball player takes a right-handed batting stance but is left-eye dominant, or uncrossed with preservation of dominance within a side [5]. As such, laterality preferences in sports reflect a complex combination of sports-specific states and population-level traits that may offer relative advantages to some individuals at the expense of others.

One theory used to explain the relatively higher left-sided 
prevalence in athletes is that left-handedness is associated with benefits in the form of a "fighting advantage," or infrequent exposure of the opponent to left-handed strategies $[6,4,7,8]$. Professional rankings of athletes from both dynamic (where athletes track multiple objects, e.g., teammates, opponents, and a ball) and interceptive sports (where athletes must manually intercept a moving target) have consistently found over-representation of left-handers, whereas no such bias exists in static sports [9, 10, 11], suggesting a competitive advantage for left-handed players [12]. Another interesting pattern has been observed in studies examining crossed vs. uncrossed dominance, particularly in static sports. These studies generally report greater performance in uncrossed (i.e., hand and eye) participants, which likely facilitates the process of aiming [13].

Despite progress towards understanding the manifestation of laterality preferences in athletes, the current literature is based on studies with relatively small sample sizes $[9,14]$ or observational data conducted in different sporting populations [15, 16, 17]. Most importantly, there lacks a comprehensive and comparative examination of eye, hand, and foot preference among athletes of different levels and primary sports. To begin addressing this need, the current study reports secondary data analysis on sensorimotor evaluations performed with Nike Sensory Station.

Between 2011 and 2015, Nike Sensory Stations were used to quantitatively evaluate visual and motor skills with goals of deriving sport-specific normative information and providing a platform for sensorimotor assessment and training. In about 25-minutes, participants completed a registry of information about themselves, including their sport, position, and competition level in order to derive group norms, along with a battery of 9 sports-relevant, visual-motor tasks administered under standardized conditions with video instructions. In addition, self-reported hand and foot preference for the athletes' primary sport of participation were registered along with eye dominance measured using the Miles Test. Data from these assessments were maintained on a central database and used to provide feedback to individuals about their relative abilities and to monitor learning coupled with sensorimotor training interventions.

Given current gaps in knowledge about laterality preferences in athletes, the current study compares eye, hand, and foot laterality preferences (including cross dominance) in a large sample of athletes from different sports and competition levels.

\section{Methods}

\subsection{Participants}

The present sample of 1770 athletes was selected from a larger database of individuals evaluated with the Nike Sensory Station. This sample includes assessments collected on individuals who self-reported as male participants in one of five primary sports: American football, baseball, basketball, ice hockey, and soccer. These assessments were collected from 26 centers worldwide between April 2010 and
December 2014. Females were omitted due to relatively small or empty cells in several sport-by-level combinations. In addition, male athletes from sports with small sample sizes were excluded to focus on sports with substantial representation in each sport-by-level combination (all individual cells $>36$ individuals).

Data were included in subsequent analyses for participants listed as middle school athletes between the ages of 10 and 16, high school athletes between 14 and 19, collegiate athletes between 17 and 28, or professional athletes between 18 and 40. This resulted in the inclusion of 200 middle school, 337 high school, 513 collegiate, and 720 professional athletes. For analysis purposes, the middle and high school athletes combined into a single "middle/high school" sample allowing for sufficient representation in all sport-by-level combinations. Across the five sports, 682 individuals listed baseball as their primary sport, 544 listed football, 235 listed soccer, 177 listed ice hockey, and 132 listed basketball. The mean age across the full sample was 21.9 years (4.9 SD) and the mean height was 71.2 inches (4.9 SD). Within this sample, 485 participants reported having experienced at least 1 concussion, with only $0.6 \%$ of the sample reporting a concussion sustained within the last month. All participants reported normal or corrected-to-normal vision at testing.

All data were shared with the research team under a secondary-data protocol approved by the Duke University Institutional Review Board [IRB B0706]. Under this protocol all data was collected for "real world use" without informed consent and shared with the research team after removal of protected health information (PHI). As such, these data conform to U.S. Department of Health and Human Services, "Regulatory considerations regarding classification of projects involving real world data" [18].

\subsection{Assessments}

All individuals were evaluated on Nike Sensory Stations, which records demographic information about participants as well as nine behavioral tasks. For this report, only demographic information is considered.

Each participant registered a demographic profile including personal information (gender, age, height), sport information (primary sport, position, level), and relevant medical history (vision correction, concussions). This section also included self-reported hand and foot preference in their main sporting activity (left, right, ambidextrous/ambipedal). Eye dominance was evaluated separately using the Miles Test, which asked individuals to place one hand over the other, raise their hands in front of their face, and peer through the gap between thumbs and index fingers at the experimenter's nose. The participant eye that was centered from the experimenter's perspective was noted. This procedure was repeated three more times. The participant's eye that was centered on the majority of trials was deemed the dominant eye. If the participant centered each eye twice, then the participant was listed as ambiocular.

Following entry of the demographic profile, nine behavioral tasks were completed in the following fixed order: 
Visual Clarity, Contrast Sensitivity, Depth Perception, NearFar Quickness, Target Capture, Perception Scan, Eye-Hand Coordination, Go/No-Go, and Response Time. Variability in task performance has been reported elsewhere [19, 20, 21, $22,23,24,25]$ and is not considered in this report.

\subsection{Data Analysis}

To evaluate laterality associations, Chi-Square tests were performed. Preliminary analyses used three-level contrasts: left, right, and ambi preference. However, when tested in relation to other variables-of-interest, greater than $20 \%$ of expected cell counts were less than 5, therefore violating fundamental Chi-Square assumptions [26]. As such, to assess the relationships between laterality preferences, Athlete Level, and Primary Sport, Chi-Square tests were performed for each pairwise contrast excluding the ambidextrous respondents. These tests were examined in an analysis for linear trends (Mantel-Hansel, Chi-Square test) with odds ratios calculated to determine the likelihood that laterality associations differed between different athlete levels and primary sport. To determine the source of laterality differences, post-hoc ChiSquare tests compared each Athlete Level by Primary Sport cell with the sum of all other cells. Statistical analysis was performed using SPSS for Windows, Release 21.

\section{Results}

\subsection{Laterality Preferences}

As illustrated in Table 1, the vast majority of the 1645 athlete participants - excluding ambidextrous $(\mathrm{N}=29)$, ambipedal $(\mathrm{N}=28)$, and ambiocular $(\mathrm{N}=73)$ responders reported right-sided hand, foot, and eye preferences. Proportionally, this right-sided majority was large for handedness $(88.6 \%)$ and footedness $(84.3 \%)$ and smaller for eye dominance $(66.0 \%)$. The percentage of right-hand dominance decreased as a function of athlete level, from middle/high school $(91.20 \%)$ to college $(90.40 \%)$ to professional $(85.30 \%)$. A similar pattern was observed for right-foot $(89.00 \%$ to $84.70 \%$ to $80.40 \%)$ and right-eye $(69.40 \%$ to $68.00 \%$ to $61.90 \%)$ dominance.

Table 1. Numbers of athletes exhibiting each pairwise combination of eye, hand, and foot preferences, excluding ambidextrous, ambipedal, and ambiocular individuals.

\begin{tabular}{|c|c|c|c|c|c|}
\hline & & & & Hanc & \\
\hline & & & & Left & Right \\
\hline \multirow{4}{*}{ Eye } & \multirow{2}{*}{ Left } & \multirow{2}{*}{ Foot } & Left & 65 & 57 \\
\hline & & & Right & 23 & 415 \\
\hline & \multirow{2}{*}{ Right } & \multirow{2}{*}{ Foot } & Left & 59 & 78 \\
\hline & & & Right & 41 & 907 \\
\hline
\end{tabular}

To evaluate the consistency of laterality preferences across body parts, cross dominance was calculated for each pairwise hand, foot, and eye combination, as well as across all three pairings (Table 2). All four of these contrasts revealed greater occurrences of uncrossed, relative to crossed, dominance. In particular, hand-foot preferences demonstrated the highest hemispheric consistency (i.e., uncrossed, both left or both right preference), with $87.9 \%$ of respondents reporting uncrossed preferences.

Table 2. Prevalence of cross dominance for hand-foot, hand-eye, eye-foot, and eye-foot-hand excluding ambidextrous, ambipedal, and ambiocular individuals.

\begin{tabular}{llllll}
\hline & & Hand-Foot & Hand-Eye & Eye-Foot & 3 Cross \\
\hline \multirow{2}{*}{ Athletes } & Crossed & $199(12.1 \%)$ & $572(34.8 \%)$ & $575(35.0 \%)$ & $673(40.9 \%)$ \\
& Uncrossed & $1446(87.9 \%)$ & $1073(65.2 \%)$ & $1070(65.0 \%)$ & $972(59.1 \%)$ \\
\hline
\end{tabular}

Chi-Square analysis indicated that there were significant hand-foot associations for both left- $\left(\chi^{2}=166.18\right.$, $\mathrm{df}=1, \mathrm{p}$ $<.001)$ and right- $\left(\chi^{2}=214.71, \mathrm{df}=1, \mathrm{p}<.001\right)$ eye dominant samples (i.e., the relationship between hand and foot preference differed from its expected values). This difference was driven by greater-than-expected left-left and right-right (i.e., uncrossed) preference, consistent with the descriptive report of crossed/uncrossed dominance above. On the other hand, hand-eye associations were not significant for either left $\left(\chi^{2}=2.70, \mathrm{df}=1, \mathrm{p}=.10\right)$ or right $\left(\chi^{2}=.58, \mathrm{df}=1, \mathrm{p}=.45\right)$ foot dominant samples. Lastly, foot-eye associations were significant for both left- $\left(\chi^{2}=4.61, \mathrm{df}=1, \mathrm{p}=.03\right)$ and right$\left(\chi^{2}=6.56, \mathrm{df}=1, \mathrm{p}<.01\right)$ foot dominant samples, driven by a larger-than-expected count of uncrossed preference.

\subsection{Laterality Preferences as a Function of Athlete Level and Primary Sport}

To test if laterality associations differed as a function of either Athlete Level and/or Primary Sport, Chi-Squared odds ratios were calculated for the three level and five sport subgroups. Tests of Homogeneity of the Odds Ratio indicated a significant difference in hand-foot associations (excluding ambidextrous and ambipedal athletes, $\mathrm{N}=1715)$ between the three Athlete levels and the five Primary Sports $\left(\chi^{2}=37.08, \mathrm{df}=14, p=.001\right)$.

Table 3. Post-hoc paired contrasts of each athlete level by primary sport cell compared to the sum of all other cells. Professional baseball players were the only group to significantly differ. * indicates significant difference (corrected for multiple comparisons, $p<.003$ ), while $†$ indicates uncorrected significance $(p<.05)$.

\begin{tabular}{llll}
\hline Group vs. Others & & $\chi^{2}$ & p-value \\
\hline \multirow{3}{*}{ Baseball } & Middle/High & 0.74 & .39 \\
& College & 0.15 & .70 \\
& Pro* & 29.63 & $<.001$ \\
Football & Middle/High & 0.73 & .39 \\
& College & 0.89 & .35 \\
& Pro & 0.22 & .64 \\
Soccer & Middle/High & 1.34 & .25 \\
& College & 0.03 & .86 \\
& Pro & 0.84 & .36 \\
Basketball & Middle/High & 0.97 & .33 \\
& College† & 4.79 & .03 \\
& Pro & 0.18 & .68 \\
Hockey & Middle/High & 2.97 & .09 \\
& College & 0.20 & .65 \\
\hline
\end{tabular}


To further evaluate this difference, Athlete Level by Primary Sport cells were compared with the sum of all other cells (e.g., college baseball athletes vs. all other athletes). As shown in Table 3, this analysis revealed that professional

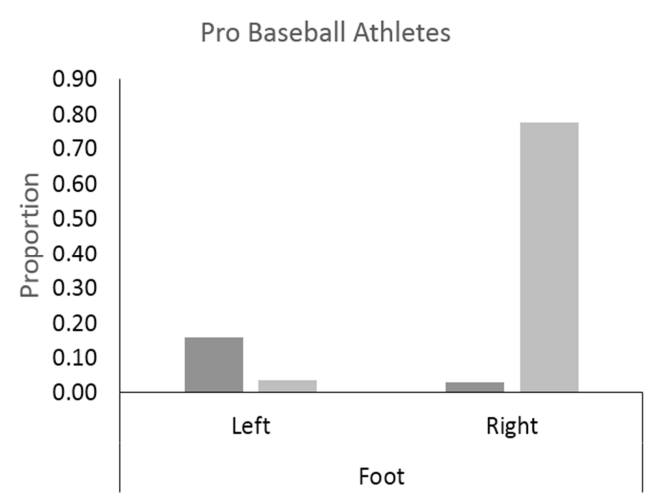

baseball players were the only group to significantly differ after controlling for multiple comparisons. As shown in Figure 1, professional baseball players exhibited a greater proportion of left-handed athletes and left-footed athletes.

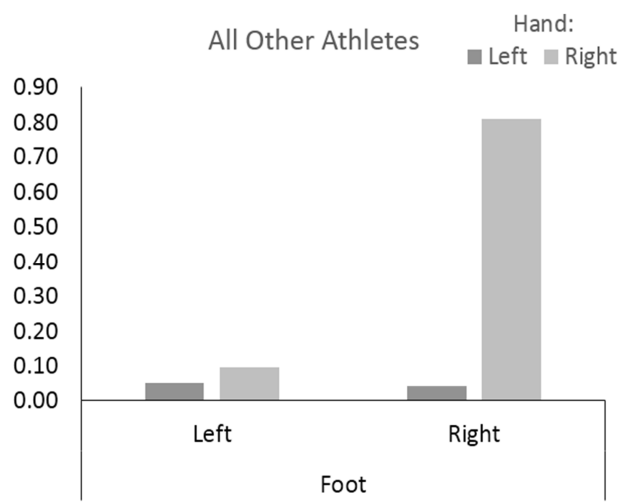

Figure 1. Pro baseball athletes exhibited a greater proportion of left-hand and left-foot dominance relative to all other athletes.

To further illuminate whether positional demands account for the greater proportion of left-handed and left-footed athletes in pro baseball, Chi-Squared odds ratios were calculated between pitchers $(\mathrm{N}=176)$ and hitters $(\mathrm{N}=201)$. Tests of Homogeneity of the Odds Ratio, however, did not reveal a significant difference between these positions $\left(\chi^{2}=\right.$ $1.17, \mathrm{df}=1, p=.28)$.

In contrast to the hand-foot preferences, hand-eye preferences (excluding ambidextrous and ambiocular athletes, $\mathrm{N}=1670$ ) did not differ significantly between the three Athlete Levels and five Primary Sports $\left(\chi^{2}=12.67, \mathrm{df}=\right.$ 14, $\mathrm{p}=.55$ ). Similarly, foot-eye preferences (excluding ambipedal and ambiocular athletes, $\mathrm{N}=1669$ ) also did not differ significantly between the three Athlete Levels and five Primary Sports $\left(\chi^{2}=12.37, \mathrm{df}=14, \mathrm{p}=.58\right)$.

\section{Discussion}

This study examined laterality preferences in a large cohort of 1770 athletes spanning from middle schoolers to professionals, across five heavily-represented sports: baseball, basketball, football, hockey, and soccer, comprising secondary analysis of real-world data collected on Nike Sensory Stations, therefore allowing for the descriptive evaluation of self-reported and measured laterality preferences. The key results observed in this study are discussed below along with the importance, challenges, and limitations inherent in characterizing and interpreting laterality preferences.

Across the 1645 athletes who did not report ambilateral preferences in our sample, a general pattern of lateral conservation was observed for hand-foot and eye-foot, but not eye-hand, preferences. Consistent with past reports [9, $10,7]$, a larger proportion of right-sided preference were seen for all categories, as well as a larger proportion of uncrossed relative to crossed dominance. The observed uncrossed handfoot preference supports typical reports of dominance in one cerebral hemisphere [27]. Conversely, ocular dominance is typically not found to correlate with other types of lateral body dominance [28], though past research suggests gender differences potentially due to environmental pressures for males, possibly contributing to the pattern of results observed here [29]. Given that past works investigating relationships between laterality associations and on-field performance have been equivocal $[30,5]$, future research may wish to further investigate the relationships between crossed/uncrossed dominance and sporting achievement.

As a function of Athlete Level and Primary Sport, a larger proportion of left-handed and left-footed athletes was seen in professional baseball. This difference echoes the conventional wisdom that left hand preference is advantageous in baseball, [15, 31, 16] which is often attributed to either structural advantages (e.g. left hand batters are closer to first base and have a better viewing angle on pitches delivered by right-handed pitchers, who make up $\sim 75 \%$ of their competition) or to prevalence advantages (e.g. greater familiarity with right-handed pitchers). The left-sided preference in the current data did not appear to be driven by differences between pitchers and hitters. Previous studies have not reported greater left-foot preference in baseball; future work should seek to replicate this finding and investigate whether it confers a competitive advantage. It is worth noting that equivalent left-sided, hand-eye and footeye associations were not observed for baseball, and no other associations were present for any other sport at any level.

Past research with Nike Sensory Stations has provided insight into the sensorimotor capacities of athletes [24, 25] and non-athletes [19, 21, 23], and has been used to test the efficacy of sports training interventions [22]. Performance variability on these assessments has been linked to on-ice performance in collegiate hockey players [20] and on-field performance in professional baseball players [25], as well as the occurrence of head impacts in collegiate football players $[32,33]$. Collectively, this research suggests that this battery may serve as a useful tool for understanding human performance [34], while also warranting further investigation into the impacts sensorimotor characteristics have on athlete performance outcomes. 
Finally, it is worth noting several limitations related to the current investigations. To begin, it is important to reiterate that this study consisted of a secondary analysis of real-world data and thus did not implement a balanced design such that all participant groups entailed equal representation. Because of the relative frequency of each gender in the database available for analysis, this investigation focuses on males. Since gender differences are known to influence laterality preferences [29], future research may wish to evaluate laterality differences in female athletes in greater depth. Importantly, past research has demonstrated that laterality preferences are not a simple binary demarcation [35] and that different types of dominance measures (e.g. sensory, sighting, and persistence in binocular rivalry for vision) are often not in agreement [28]. As such, the current binary demarcations likely reflect an oversimplification of dominance preferences. Moreover, the distinction between sport-specific and general laterality may be in opposition, particularly in ice hockey. It is not uncommon for ice hockey players to be generally right-handed, but play hockey lefthanded (and vice versa) because it is conventional to use the dominant hand on the top of the stick. As such, there may be operational disparity within the report of laterality preferences. Nonetheless, the current study is notable in light of the considerable sample size and the fact that laterality preferences are quantitatively compared to psychometrically measured sensorimotor abilities.

\subsection{Conclusion}

By examining laterality preferences in a large cohort of 1770 athletes, the current study provides a descriptive evaluation of self-reported and measured laterality preferences. Results showed a pattern of lateral conservation (uncrossed dominance) for hand-foot and eye-foot, but not eye-hand, preferences. Moreover, group differences were observed with a higher proportion of left-hand and left-foot preferences in professional baseball players, supporting the notion that left hand preference is advantageous in baseball.

\section{Funding}

This research was funded by grant support to L. G. A. through the United States Army Research Office [W911NF15-1-0390].

\section{Conflict of Interest Statement}

The authors do not have any commercial, proprietary or financial interest in any device, equipment, instrument, or drug related to this article.

\section{Acknowledgements}

The authors would like to thank Ian Muir, Herb Yoo, and Fred Gregory for their assistance in the implementation of this research project, and Ms. Susan Hilbig for her valuable input on the manuscript.

\section{References}

[1] Carey, D. P. and C. V. Hutchinson (2013). "Looking at eye dominance from a different angle: is sighting strength related to hand preference?" Cortex 49 (9): 2542-2552.

[2] Loffing, F., F. Solter and N. Hagemann (2014). "Left preference for sport tasks does not necessarily indicate lefthandedness: sport-specific lateral preferences, relationship with handedness and implications for laterality research in behavioural sciences." PLoS One 9 (8): e105800.

[3] Llaurens, V., M. Raymond and C. Faurie (2009). "Why are some people left-handed? An evolutionary perspective." Philos Trans R Soc Lond B Biol Sci 364 (1519): 881-894.

[4] Abrams, D. M. and M. J. Panaggio (2012). "A model balancing cooperation and competition can explain our righthanded world and the dominance of left-handed athletes." J R Soc Interface 9 (75): 2718-2722.

[5] Portal, J. M. and P. E. Romano (1998). "Major review: ocular sighting dominance: a review and a study of athletic proficiency and eye-hand dominance in a collegiate baseball team." Binocul Vis Strabismus Q 13 (2): 125-132.

[6] Faurie, C., V. Llaurens, A. Alvergne, M. Goldberg, M. Zins and M. Raymond (2011). "Left-handedness and male-male competition: insights from fighting and hormonal data." Evol Psychol 9 (3): 354-370.

[7] Loffing, F., J. Schorer, N. Hagemann and J. Baker (2012). "On the advantage of being left-handed in volleyball: further evidence of the specificity of skilled visual perception." Attention, Perception, \& Psychophysics 74 (2): 446-453.

[8] Faurie, C. and M. Raymond (2013). "The fighting hypothesis as an evolutionary explanation for the handedness polymorphism in humans: where are we?" Ann N Y Acad Sci 1288: 110-113.

[9] Raymond, M., D. Pontier, A. B. Dufour and A. P. Moller (1996). "Frequency-dependent maintenance of left handedness in humans." Proc Biol Sci 263 (1377): 1627-1633.

[10] Grouios, G. (2004). "Motoric dominance and sporting excellence: training versus heredity." Percept Mot Skills 98 (1): 53-66.

[11] Loffing, F. and N. Hagemann (2012). Side bias in human performance: A review on the left-handers' advantage in sports. Bias in Human Behaviour. M. M. Dutta T, Kumar S.. Hauppauge, NY, Nova Science: 163-182.

[12] Loffing, F. and N. Hagemann (2015). "Pushing through evolution? Incidence and fight records of left-oriented fighters in professional boxing history." Laterality: Asymmetries of Body, Brain and Cognition 20 (3): 270-286.

[13] Sheeran, T. J. (1985). "Effect of pure and crossed dextrality on marksmanship skill." Perceptual and motor skills 61 (3_suppl): 1171-1174.

[14] Carey, D. P., G. Smith, D. T. Smith, J. W. Shepherd, J. Skriver, L. Ord and A. Rutland (2001). "Footedness in world soccer: an analysis of France'98." Journal of Sports Sciences 19 (11): 855-864. 
[15] Goldstein, S. R. and C. A. Young (1996). "' Evolutionary" stable strategy of handedness in major league baseball." Journal of Comparative Psychology 110 (2): 164.

[16] Clotfelter, E. D. (2008). "Frequency-dependent performance and handedness in professional baseball players (Homo sapiens)." Journal of Comparative Psychology 122 (1): 68.

[17] Loffing, F. and N. Hagemann (2016). Performance differences between left-and right-sided athletes in one-on-one interactive sports. Laterality in Sports, Elsevier: 249-277.

[18] DHHS (2015). Secretary's Advisory Committee on Human Research Protections. Attachment A: human subjects research implications of "big data" studies. U. S. D. o. H. a. H. Services. 2016

[19] Erickson, G. B., K. Citek, M. Cove, J. Wilczek, C. Linster, B. Bjarnason and N. Langemo (2011). "Reliability of a computer-based system for measuring visual performance skills." Optometry 82 (9): 528-542.

[20] Poltavski, D. and D. Biberdorf (2015). "The role of visual perception measures used in sports vision programmes in predicting actual game performance in Division I collegiate hockey players." J Sports Sci 33 (6): 597-608.

[21] Wang, L., K. Krasich, T. Bel-Bahar, L. Hughes, S. R. Mitroff and L. G. Appelbaum (2015). "Mapping the structure of perceptual and visual-motor abilities in healthy young adults." Acta Psychol (Amst) 157: 74-84.

[22] Appelbaum, L. G., Y. Lu, R. Khanna and K. Detwiler (2016). "The Effects of Sports Vision Training on Sensorimotor Abilities in Collegiate Softball Athletes." Athletic Training \& Sports Health Care.

[23] Krasich, K., B. Ramger, L. Holton, L. Wang, S. R. Mitroff and L. Gregory Appelbaum (2016). "Sensorimotor Learning in a Computerized Athletic Training Battery." J Mot Behav 48 (5): 401-412.

[24] Klemish, D., B. Ramger, K. Vittetoe, J. P. Reiter, S. Tokdar and L. G. Appelbaum (2017). "Visual Abilities Distinguish Pitchers from Hitters in Professional Baseball.." Journal of Sports Sciences.

[25] Burris, K., K. Vittetoe, B. Ramger, S. Suresh, S. T. Tokdar, J. P. Reiter and L. G. Appelbaum (2018). "Eye on the ball: The relationship between sensorimotor abilities and on-field performance in professional baseball." Nature Scientific Reports. 8 (116).

[26] Yates, D., D. Moore and G. McCabe (1999). The Practice of Statistics. New York, W. H. Freeman.

[27] Sadeghi, H., P. Allard, F. Prince and H. Labelle (2000). "Symmetry and limb dominance in able-bodied gait: a review." Gait Posture 12 (1): 34-45.

[28] Suttle, C., J. Alexander, M. Liu, S. Ng, J. Poon and T. Tran (2009). "Sensory ocular dominance based on resolution acuity, contrast sensitivity and alignment sensitivity." Clin Exp Optom 92 (1): 2-8.

[29] Porac, C. and S. Coren (1975). "Is eye dominance a part of generalized laterality?" Percept Mot Skills 40 (3): 763-769.

[30] Laby, D. M., D. G. Kirschen, A. L. Rosenbaum and M. F. Mellman (1998). "The effect of ocular dominance on the performance of professional baseball players." Ophthalmology 105 (5): 864-866.

[31] Grondin, S., Y. Guiard, R. B. Ivry and S. Koren (1999). "Manual laterality and hitting performance in major league baseball." J Exp Psychol Hum Percept Perform 25 (3): 747754.

[32] Harpham, J. A., J. P. Mihalik, A. C. Littleton, B. S. Frank and K. M. Guskiewicz (2014). "The effect of visual and sensory performance on head impact biomechanics in college football players." Ann Biomed Eng 42 (1): 1-10.

[33] Asken, M. B., J. P. Milhalik, J. D. Schmidt, A. C. Littlleton, K. M. Guskiewicz and J. B. Hopfinger (2016). "Visual performance measures and functional implications in healthy participants: A sports concussion perspective." Athletic Training \& Sports Health Care 8 (4): 145-153.

[34] Appelbaum, L. G. and G. B. Erickson (2016). "Sports vision training: a review of the state-of-the-art in digital training techniques." International Review of Sport and Exercise Psychology Published on line, Nov 23, 2016: 1-30.

[35] Annett, M. (1994). "Handedness as a continuous variable with dextral shift: sex, generation, and family handedness in subgroups of left- and right-handers." Behav Genet 24 (1): 5163. 